\title{
Hopping conduction in size-controlled Si nanocrystals
}

\author{
M. A. Rafiq \\ Microelectronics Research Centre, Cavendish Laboratory, University of Cambridge, Madingley Road, \\ Cambridge CB3 OHE, United Kingdom and SORST, Japan Science and Technology (JST)
}

Y. Tsuchiya, H. Mizuta, and S. Oda

Department of Physical Electronics, Tokyo Institute of Technology, O-Okayama, Meguro-ku, Tokyo 152-8552, Japan and SORST, Japan Science and Technology (JST)

\author{
Shigeyasu Uno \\ Department of Electrical Engineering and Computer Science, Graduate School of Engineering, \\ Nagoya University, Furo-cho, Chikusa-ku, Nagoya 464-8603, Japan and SORST, \\ Japan Science and Technology (JST)
}

\section{Z. A. K. Durrania) and W. I. Milne}

Electronic Devices and Materials Group, Engineering Department, University of Cambridge, Trumpington Street, Cambridge CB2 1PZ, United Kingdom and SORST, Japan Science and Technology (JST)

(Received 31 March 2006; accepted 24 April 2006; published online 7 July 2006)

\begin{abstract}
We investigate the temperature dependence of conduction in size-controlled silicon nanocrystals. The nanocrystals are $\sim 8 \mathrm{~nm}$ in diameter, covered by $\sim 1.5 \mathrm{~nm}$ thick $\mathrm{SiO}_{2}$ shells. In $300 \mathrm{~nm}$ thick films for temperatures $T$ from 30 to $200 \mathrm{~K}$, the conductivity $\sigma$ follows a $\ln (\sigma) \mathrm{vs} 1 / T^{1 / 2}$ dependence. This may be associated with either percolation-hopping conductance or Efros-Shklovskii variable range hopping. Assuming hopping sites only on the nanocrystals, the data agree well with the percolation model. (C) 2006 American Institute of Physics. [DOI: 10.1063/1.2209808]
\end{abstract}

\section{INTRODUCTION}

Densely packed arrays of semiconductor nanocrystals form another class of "nanocomposite" materials, with different electronic and optical properties. Various semiconductor-based nanocomposites have now been prepared, e.g., $\mathrm{Si}, \mathrm{Ge}, \mathrm{CdSe}, \mathrm{PbSe}$, or $\mathrm{ZnO}$ nanocrystals dispersed in a $\mathrm{SiO}_{2}, \mathrm{Si}_{3} \mathrm{~N}_{4}$, or polymer-based insulating matrix. $^{1-7}$ The properties of these materials result from strong quantum confinement and single-electron charging of individual particles and interaction between neighboring particles through these effects. ${ }^{8}$ There is considerable interest in the application of these materials to single-electron transistors, ${ }^{9}$ nonvolatile memories, ${ }^{10}$ electron emitters, ${ }^{11}$ and optoelectronic devices. $^{1,7}$ In addition, Si nanocrystals are of great interest in the development of nanoscale devices for integrated circuit applications.

In investigations of the conduction mechanism in semiconductor nanocrystals, hopping and Coulomb charging transport mechanisms have been observed. ${ }^{2,4,6}$ However, there are often large variations in the nanocrystal size and separation, complicating analysis of the data. Furthermore, there are only limited investigations of the transport properties of $\mathrm{Si}$ nanocrystals. A space charge limited current (SCLC) has been observed at temperatures $>200 \mathrm{~K}$ in $\mathrm{Si}$ nanocrystals prepared by plasma decomposition of silane. ${ }^{12}$ Fujii et al. ${ }^{13}$ have investigated $\sim 2 \mathrm{~nm}$ Si clusters dispersed in $\mathrm{SiO}_{2}$ films, formed by sputtering $\mathrm{Si}$ into the $\mathrm{SiO}_{2}$ films. They observe that the conductivity $\sigma$ follows a $\ln (\sigma)$ vs $1 / T^{1 / 4}$ temperature dependence from 120 to $300 \mathrm{~K}$, attributable to Mott variable range hopping (M-VRH).

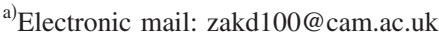

In this paper, we investigate the conduction mechanism at temperatures $<200 \mathrm{~K}$ in Si nanocrystals $\sim 8 \mathrm{~nm}$ in size, with controlled size and separation. We observe a $\ln (\sigma)$ vs $1 / T^{1 / 2}$ dependence over a wide range of temperature $(30-200 \mathrm{~K})$, which can be attributed to either the percolation-hopping conduction model of Šimánek ${ }^{14}$ or to Efros-Shklovskii variable range hopping (ES-VRH), where Coulomb interactions are considered in the hopping process. ${ }^{15}$ Assuming hopping sites only on the nanocrystals, our data fit well with the percolation-hopping model.

\section{DEVICE FABRICATION}

The measurements were performed using Al/Si nanocrystal film/ $p$-Si/Al diodes, similar to our previous work on SCLC in $\mathrm{Si}$ nanocrystals. ${ }^{12}$ The nanocrystal film was $\sim 300 \mathrm{~nm}$ thick, deposited at room temperature by plasma decomposition of $\mathrm{SiH}_{4}$ Ref. 1 on $p$-type $\mathrm{Si}$ substrates with a resistivity of $10.0 \Omega \mathrm{cm}$. The nanocrystals were $8 \pm 1 \mathrm{~nm}$ in diameter and crystalline in structure, with $\sim 1.5 \mathrm{~nm}$ surface $\mathrm{SiO}_{2}$ shells. For nanocrystals near the base of the film, the oxide thickness is likely to be somewhat less. Figure 1 shows a scanning electron micrograph of the film. The left inset to the figure shows a schematic of the film structure. The nanocrystal coverage is $\sim 60 \%$ /layer, and voids in the film do not span the film thickness. The devices consisted of mesas defined using e-beam lithography and reactive ion etching with an $\mathrm{Al}$ etch mask. The top contact to the nanocrystals and the back contact to the substrate were formed by $300 \mathrm{~nm}$ thick Al layers. The device area, determined by the top contact, varied from $35 \times 35 \mu \mathrm{m}^{2}$ to $200 \times 200 \mu \mathrm{m}^{2}$. 


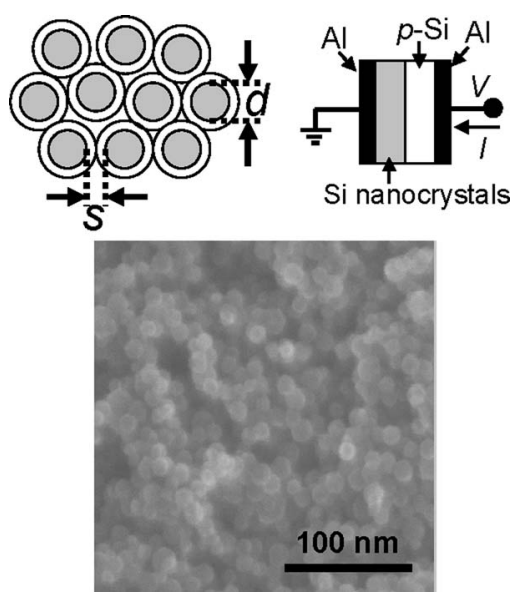

FIG. 1. Scanning electron micrograph of the Si nanocrystal film. Left inset: Schematic of nanocrystal film structure, with nanocrystal size $d$ and the nanocrystal separation $s$. Right inset: Schematic of $\mathrm{Al} / \mathrm{Si}$ nanocrystal/ $p$-Si/ $\mathrm{Al}$ diode, with measurement configuration.

\section{RESULTS AND DISCISSION}

Figure 2 shows the $I-V$ characteristics for an $\mathrm{Al} / \mathrm{Si}$ nanocrystal $/ p$-Si/Al diode of area of $35 \times 35 \mu \mathrm{m}^{2}$, from 200 to $40 \mathrm{~K}$ in steps of $20 \mathrm{~K}$. The characteristics were measured using a cryogenic temperature prober (Nagase \& Co., Ltd.) with a base temperature of $\sim 20 \mathrm{~K}$ and an Agilent 4156A parameter analyzer. The measurement configuration is shown in Fig. 1, right inset. Figure 2(a) shows the $I-V$ characteristics on a linear scale, Fig. 2(b) shows the characteristics on a log-linear scale, and Fig. 2(c) shows an Arrhen-
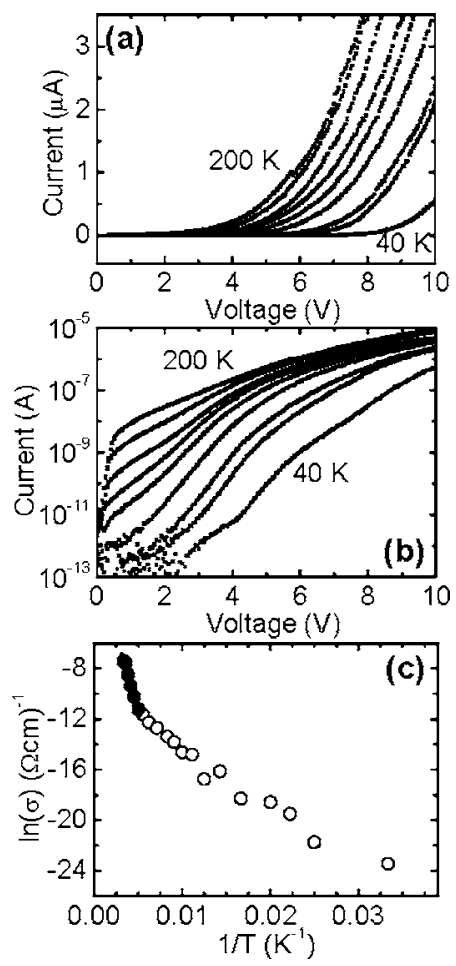

FIG. 2. (a) $I-V$ characteristics of an $\mathrm{Al} / \mathrm{Si}$ nanocrystal $/ p-\mathrm{Si} / \mathrm{Al}$ diode, from 200 to $40 \mathrm{~K}$, plotted on a linear scale. The temperature step is $20 \mathrm{~K}$. The device area is $35 \times 35 \mu \mathrm{m}^{2}$. (b) $I-V$ characteristics of (a), plotted on a $\log$-linear scale. (c) Arrhenius plot, $\ln (\sigma)$ vs $1 / T$, where $\sigma$ is the device conductivity and $T$ is the measurement temperature. The applied bias is $4 \mathrm{~V}$. ius plot of the conductance $\sigma$ at $4 \mathrm{~V}$ applied bias. These data are a subset of the data presented in our previous work, where we investigated SCLC in Si nanocrystals. ${ }^{12}$ In our previous work, we investigated a higher temperature range $(200-300 \mathrm{~K})$ than in this work and observed SCLC in the presence of an exponential distribution of traps. Here, we investigate the $I-V$ characteristics from 200 to $40 \mathrm{~K}$, where SCLC is not observed.

The $I-V$ characteristics of Figs. 2(a) and 2(b) are strongly nonlinear and are measured with a "forward" (positive) bias applied to the $p$-Si/Al back contact. Holes are then injected from the $p$-Si substrate into the nanocrystals. The current, plotted on a linear scale [Fig. 2(a)], shows a "threshold" voltage which increases from $\sim 3 \mathrm{~V}$ at $200 \mathrm{~K}$ to $\sim 8 \mathrm{~V}$ at $40 \mathrm{~K}$. The subthreshold current increases rapidly, e.g., by three orders of magnitude at $40 \mathrm{~K}$ [Fig. 2(b)].

The $p$-Si substrate in our device has a resistivity of $10 \Omega \mathrm{cm}$, very similar to the resistivity of substrates used in previous work on both $\mathrm{Si}$ nanocrystals ${ }^{2}$ and porous $\mathrm{Si}^{16}$ However, from 200 to $40 \mathrm{~K}$, the resistivity is not low enough for an Ohmic contact to form at the $\mathrm{Al} / p$-Si back contact. In this regard, we have measured the characteristics between two Al contacts to the substrate. From 200 to $40 \mathrm{~K}$, these contacts form back-to-back diodes and show an exponential increase in the current with applied bias. This implies that our $\mathrm{Al} / \mathrm{Si}$ nanocrystal $/ p-\mathrm{Si} / \mathrm{Al}$ diode consists of the nanocrystal film resistance in series with a rectifying diode formed by the back contact. In addition, a further rectifying diodelike contact may form at the nanocrystal $/ p-\mathrm{Si}$ interface. ${ }^{2}$ It is likely that the subthreshold region in Fig. 2(a) is dominated by these rectifying diodes. With an increasing forward bias (positive bias at the back contact) across the full $\mathrm{Al} / \mathrm{Si}$ nanocrystal $/ p$-Si/Al structure, the rectifying diodes become less significant and the characteristics depend on the resistance of the nanocrystal film.

An Arrhenius plot of $\sigma$ [Fig. 2(c)] at $4 \mathrm{~V}$ bias shows two distinct regimes. Above $200 \mathrm{~K}$ (black circles), a single, steep slope is observed. This can be attributed to thermally activated transport, with an activation energy of $\sim 300 \mathrm{meV}$. Below $200 \mathrm{~K}$, the data (white circles) can be fitted to a $\ln (\sigma)$ vs $T^{n}$ dependence.

Figure 3 shows a $\ln (\sigma)$ vs $1 / T^{1 / 2}$ plot at $4 \mathrm{~V}$, from 30 to $200 \mathrm{~K}$, for the device of Fig. 2. The applied bias is as low as possible without noise affecting the data. A plot of the data at $3 \mathrm{~V}$ also shows a $\ln (\sigma)$ vs $1 / T^{1 / 2}$ dependence with similar slope to the data of Fig. 3. However, for bias $<3 \mathrm{~V}$, the rectifying diodes formed at the $\mathrm{Si}$ nanocrystal $/ p-\mathrm{Si}$ and $p$-Si/Al interfaces begin to dominate and it is difficult to observe the effect of the Si nanocrystals. For higher applied bias, the temperature dependence may be reduced due to an increasing field effect ${ }^{17}$ and here, we attempt to minimize this by not applying high voltages.

The dependence in Fig. 3 is a straight line with a slope of $111 \mathrm{~K}^{1 / 2}$. The inset shows data from a second device of $65 \times 65 \mu \mathrm{m}^{2}$ area, at $4 \mathrm{~V}$ bias. These data also follow a $\ln (\sigma)$ vs $1 / T^{1 / 2}$ dependence with a very similar slope, in this case $90 \mathrm{~K}^{1 / 2}$. We have also measured the temperature dependence 


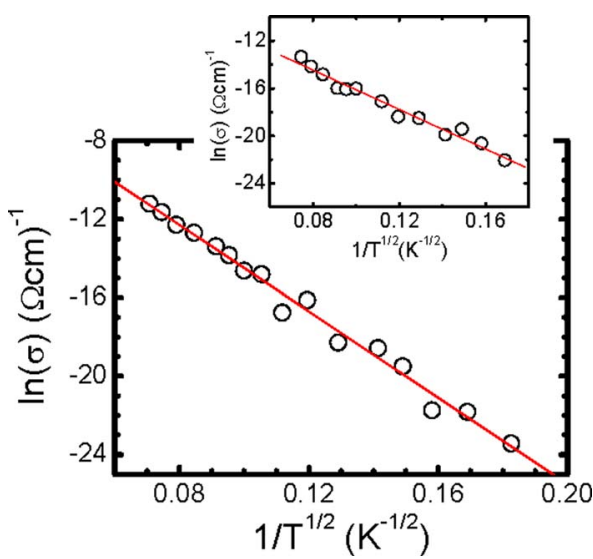

FIG. 3. $\ln (\sigma)$ vs $1 / T^{1 / 2}$ plot at $4 \mathrm{~V}$, from 30 to $200 \mathrm{~K}$, for the device of Fig. 2. The inset shows a similar plot for a second device of $65 \times 65 \mu \mathrm{m}^{2}$ area, at $4 \mathrm{~V}$ and from 35 to $200 \mathrm{~K}$. Solid lines are least square fits to the data.

of the conductivity between two $\mathrm{Al}$ contacts to the $p$-Si substrate. This does not show a $\ln (\sigma)$ vs $1 / T^{1 / 2}$ dependence in the temperature range from 200 to $40 \mathrm{~K}$.

From 30 to $200 \mathrm{~K}$, the temperature dependence of $\sigma$ is described by the equation

$$
\sigma \propto \exp \left[-\left(\frac{T_{0}}{T}\right)^{1 / 2}\right] .
$$

Here, $T_{0}$ is a constant of the material and can be determined from the slope of the $\ln (\sigma)$ vs $1 / T^{1 / 2}$ plot. This behavior is commonly observed in (i) metal-insulator composite films ${ }^{14,18}$ and (ii) amorphous or doped semiconductor materials. ${ }^{15,19}$ Our films are similar to the former group. Two carrier transport mechanisms may explain the temperature dependence. For metal-insulator composites, Šimánek ${ }^{14}$ has proposed a percolation-hopping transport model. Alternatively, in both groups of materials, an ES-VRH model may be valid. ${ }^{15}$

The percolation-hopping transport model, where thermally activated carrier tunneling between adjacent nanocrystals, i.e., nearest neighbor hopping, is considered over a wide temperature range, has been applied to Ge nanocrystals in $\mathrm{SiO}_{2}{ }^{4}$ The nanocrystal size $d$ and separation $s$ (Fig. 1, left inset) are regarded as random, uncorrelated variables. The hopping activation energy $E_{a}$ is taken to be the energy difference between the first electron levels $E_{1}$ on neighboring nanocrystals. Here, $E_{1}=E_{c}+E_{d}$, where $E_{c}$ is the singleelectron charging energy and $E_{d}$ is the quantum confinement energy.

For nanocrystals in an insulating matrix, we approximate $E_{c}=\left(e^{2} / 4 \pi \varepsilon \varepsilon_{0} d\right)\{(s / d) /[(1 / 2)+(s / d)]\}$, where $\varepsilon$ is the matrix dielectric constant and $\varepsilon_{0}$ is the permittivity of free space. In addition, $E_{d}=\hbar^{2} \pi^{2} / 2 m^{*}(d / 2)^{2}$, where $m^{*}$ is the carrier effective mass. Any variation in $s$ and $d$ leads to variation in $E_{1}$ and therefore in $E_{a}$. As the resistance between the nanocrystals varies strongly with $E_{a}$, the nanocrystal film can be modeled as a three-dimensional random resistor network, described by a critical percolation conductance. Šimánek used this to obtain Eq. (1), with the material constant $T_{0}$ given by

$$
T_{0}=\frac{2 P_{c} s_{\max } E_{a, \max }}{k_{B} \alpha} .
$$

Here, $k_{B}$ is the Boltzmann constant, $P_{c}$ is the percolation threshold, $s_{\max }$ is the maximum particle separation, $E_{a, \max }$ is the maximum value of the activation energy, and $\alpha$ is the carrier wave function decay length in the insulating matrix.

For our films, $s_{\max } \approx 3 \mathrm{~nm}$ and $d$ varies from 7 to $9 \mathrm{~nm}$. Assuming hole transport in our forward bias data, $E_{1}$ then has a maximum value $E_{1, \max }=E_{c, \text { max }}+E_{d, \text { max }} \approx 118 \mathrm{meV}$ and a minimum value $E_{1, \min }=E_{c, \text { min }}+E_{d, \text { min }} \approx 52 \mathrm{meV}$, leading to $E_{a, \text { max }}=E_{1, \max }-E_{1, \min }=66 \mathrm{meV}$. Here, we use an average hole effective mass $m^{*} \approx 0.57 m_{0},{ }^{20}$ where $m_{0}$ is the electron mass. Our value of $E_{a \text {, max }}$ is large compared to $k_{B} T$ for the temperature range of our data, confirming the validity of the percolation approach in our temperature range. Regarding $P_{c}$, this is unknown for our disordered films. However, Ziman $^{21}$ has argued that in both ordered and disordered lattices, $P_{c}$ follows the empirical formula $P_{c} \approx 1.5 / Z$, where $Z$ is the coordination number. For typical lattices, $P_{c}$ varies from 0.12 (face-centred cubic, $Z=12$ ) to 0.39 (diamond lattice, $Z=4)$. For our films, we use an average value of $P_{c}$ $=0.25$ (corresponding to a simple cubic lattice) as an approximation. Using $\alpha \sim 0.1 \mathrm{~nm}$ for holes in $\mathrm{SiO}_{2}$, Eq. (2) gives $T_{0} \approx 1.15 \times 10^{4} \mathrm{~K}$. This is in good agreement with our experimental value, $T_{0}=1.23 \times 10^{4} \mathrm{~K}$.

We now consider the ES-VRH mechanism. Coulomb interactions associated with electron-hole pairs created during hopping cause the density of states $g_{0}$ to vanish quadratically at the Fermi energy. Furthermore, Coulomb interactions imply that $E_{a}$ is proportional to $1 / r$, where $r$ is the hopping length. This model predicts the dependence of Eq. (1), where $T_{0}$ is given by ${ }^{15}$

$$
T_{0}=\frac{2.8 e^{2}}{4 \pi \varepsilon \varepsilon_{0} k_{B} a} .
$$

For our films, using $\alpha \sim 0.1 \mathrm{~nm}$ gives $T_{0}=1.18 \times 10^{5} \mathrm{~K}$. This is an order of magnitude larger than our experimental value.

The ES-VRH model also predicts a critical temperature $T_{c}=e^{4} g_{0} \alpha / k_{B}\left(4 \pi \varepsilon \varepsilon_{0}\right)^{2}$. Above $T_{c}$, Coulomb interactions may be neglected and a transition to a M-VRH dependence occurs. For our films, we approximate $g_{0}=1 /\left\lfloor\delta E \frac{4}{3} \pi[(s\right.$ $\left.+d) / 2]^{3}\right\rfloor$, where $\delta E \sim E_{d} \approx 40 \mathrm{meV}$ is the valence band level splitting on an $8 \mathrm{~nm}$ nanocrystal and the remaining term gives the nanocrystal and shell volume. This gives $g_{0} \approx 3.5$ $\times 10^{19} \mathrm{eV}^{-1} \mathrm{~cm}^{-3}$ and $T_{c} \approx 5.5 \mathrm{~K}$, far lower than the $200 \mathrm{~K}$ maximum temperature in our dependence.

In the above analysis, the experimental value of $T_{0}$ fits well with Šimánek's percolation model but not with the ESVRH model. This is a consequence of our assumption of hopping sites only on the nanocrystals. As the carrier localization length in $\mathrm{SiO}_{2}$ is only $\sim 0.1 \mathrm{~nm}, \mathrm{ES}-\mathrm{VRH}$ with tunneling to distant sites is less likely. However, if defect states exist in the $\mathrm{SiO}_{2}$ shells, then additional hopping sites become available and ES-VRH may become more feasible. ${ }^{22}$ Such sites would increase $g_{0}$ and therefore $T_{c}$. If we take $T_{c}$ $\sim 200 \mathrm{~K}$, comparable to our observed maximum temperature, then $g_{0}=1.26 \times 10^{21} \mathrm{eV}^{-1} \mathrm{~cm}^{-3}$. This value is very high and is difficult to attribute to defect states in the $\mathrm{SiO}_{2}$ shells. 


\section{CONCLUSIONS}

In conclusion, we investigated the conduction mechanism at $<200 \mathrm{~K}$ in Si nanocrystals arrays. Our nanocrystals are $\sim 8 \mathrm{~nm}$ in diameter, covered by a $\sim 1.5 \mathrm{~nm}$ thick $\mathrm{SiO}_{2}$ shell. We observe a $\ln (\sigma)$ vs $1 / T^{1 / 2}$ dependence from 30 to $200 \mathrm{~K}$, which may be associated with either a percolation or ES-VRH model. Assuming hopping sites only on the nanocrystals, our data agree well with a percolation model.

\section{ACKNOWLEDGMENTS}

The authors acknowledge useful discussions with Professor H. Ahmed at Corpus Christi College, Cambridge and Peyman Servati at the Department of Engineering, University of Cambridge. One of the authors (M.A.R.) would like to acknowledge the support of the Cambridge Commonwealth Trust.

${ }^{1}$ Y. Kanemitsu, S. Okamoto, M. Otobe, and S. Oda, Phys. Rev. B 55 R7375 (1997).

${ }^{2}$ T. A. Burr, A. A. Seraphin, E. Werwa, and K. D. Kolenbrander, Phys. Rev. B 56, 4818 (1997).

${ }^{3}$ L. Chen, W. Chen, and F. C. Hong, Appl. Phys. Lett. 86, 193506 (2005). ${ }^{4}$ M. Fujii, O. Mamezaki, S. Hayashi, and K. Yamamoto, J. Appl. Phys. 83, 1507 (1998).
${ }^{5}$ B. O. Dabbousi, M. G. Bawendi, O. Onitsuka, and M. F. Rubner, Appl. Phys. Lett. 66, 1316 (1995).

${ }^{6}$ H. E. Romero and M. Drndic, Phys. Rev. Lett. 95, 156801 (2005).

${ }^{7}$ Q. Chen, L. S. Schadler, R. W. Siegel, and G. C. Irvin, Jr., Proc. SPIE 5222, 158 (2003).

${ }^{8}$ C. P. Collier, T. Vossmeyer, and J. R. Heath, Annu. Rev. Phys. Chem. 49, 371 (1998).

${ }^{9}$ A. Dutta, S. Oda, Y. Fu, and M. Willander, Jpn. J. Appl. Phys., Part 1 39, 4647 (2000).

${ }^{10}$ S. Tiwari, F. Rana, H. Hanafi, A. Hartstein, E. F. Crabbé, and K. Chan, Appl. Phys. Lett. 68, 1377 (1996), P. Normand et al., ibid. 83, 168 (2003).

${ }^{11}$ K. Nishiguchi, X. Zhao, and S. Oda, J. Appl. Phys. 92, 2748 (2002).

${ }^{12}$ M. A. Rafiq, Y. Tsuchiya, H. Mizuta, S. Oda, S. Uno, Z. A. K. Durrani, and W. I. Milne, Appl. Phys. Lett. 87, 182101 (2005).

${ }^{13}$ M. Fujii, Y. Inoue, S. Hayashi, and K. Yamamoto, Appl. Phys. Lett. 68, 3749 (1996).

${ }^{14}$ E. Šimánek, Solid State Commun. 40, 1021 (1981).

${ }^{15}$ A. L. Efros and B. I. Shklovskii, J. Phys. C 8, L49 (1975).

${ }^{16}$ M. Ben-Chorin, F. Möller, and F. Koch, Phys. Rev. B 49, 2981 (1994).

${ }^{17}$ D. Yu, C. Wang, B. L. Wehrenburg, and P. Guyot-Sionnest, Phys. Rev. Lett. 92, 216802 (2004).

${ }^{18}$ P. Sheng and J. Klafter, Phys. Rev. B 27, 2583 (1983).

${ }^{19}$ G.-F. Hohl, S. D. Baranovskii, J. A. Becker, F. Hensel, S. A. Quaiser, and M. T. Reetz, J. Appl. Phys. 78, 7130 (1995).

${ }^{20}$ R. A. Smith, Semiconductors, 2nd ed. (Cambridge University Press, Cambridge, 1978).

${ }^{21}$ J. M. Ziman, J. Phys. C 1, 1532 (1968).

${ }^{22}$ J. Zhang and B. I. Shklovskii, Phys. Rev. B 70, 115317 (2004). 